\title{
Uso de las Redes Sociales Digitales en el ámbito escolar universitario
}

\author{
(The Use of Digital Social Networks in University Contexts)
}

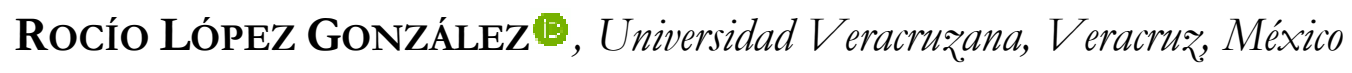

María Paulet López Flores, Universidad Veracruzana, Veracruそ, México

\section{Volumen 2, número 1}

Junio 2017

p. $25-45$

Este número se publicó el 30 de junio de 2017

ISSN: ISSN: 2448-5942, doi: https://doi.org/10.36799/el.v2i1.45

\section{Cómo citar:}

López González, R., y López Flores, M. P. (2017). Uso de las Redes Sociales Digitales en el ámbito escolar universitario. Estudios $\lambda$ ambda. Teoría y práctica de la Didáctica en Lengua y Literatura. , 2(1), 25-45. https://doi.org/10.36799/el.v2i1.45

Derechos de autor: El autor o autores conservan en todo momento sus derechos morales y patrimoniales sobre la obra; la obra no se puede alterar, transformar o ampliar; siempre debe reconocerse la autoría del documento referido. Ninguna de las modalidades de los documentos publicados en Estudios Aambda. Teoría y práctica de la didáctica en lengua y literatura tienen fines comerciales de naturaleza alguna.

Los contenidos de este artículo están bajo una licencia de Creative Commons Atribución No Comercial- Sin Derivadas 4.0 Internacional 두요 


\title{
Uso de las Redes Sociales Digitales en el ámbito escolar universitario ${ }^{1}$
}

\author{
The Use of Digital Social Networks in the University Context
}

\author{
ROCÍO LÓPEZ GONZÁLEZ ${ }^{2}$ \\ MARÍA PAULET LÓPEZ FLORES ${ }^{3}$

\section{RESUMEN}

El propósito central de este trabajo es aportar elementos de análisis que permitan conocer y comprender las actividades escolares que realizan los jóvenes universitarios a través del uso de las redes sociales digitales. Para ello, se analizaron los datos recabados a través de un cuestionario y la realización de entrevistas semiestructuradas a un grupo de jóvenes estudiantes de la Universidad Veracruzana, del sistema escolarizado, pertenecientes a diferentes áreas académicas y al campus Xalapa, cuyas edades oscilan entre 18 y 35 años. Los resultados permitieron mostrar ciertas particularidades en cuanto al uso que se les da a las redes en el ámbito escolar, incluyendo el tipo de red social que predomina, los roles en los que están inmersos, los temas, así como las formas de organización e interacción con sus pares y profesores. Se considera que este estudio brinda diversas líneas de trabajo para futuras investigaciones que permitan conocer a los jóvenes estudiantes contemporáneos que transitan por las universidades en la era de la cultura digital, que contribuyan al diseño de actividades académicas que promuevan la reflexión para una mejora del proceso de enseñanza-aprendizaje. Este trabajo fue realizado en el marco de la Red Temática Literacidad Digital en la Universidad, reconocida y aprobada por el Consejo Nacional de Ciencia y Tecnología.

PALABRAS CLAVE: Redes sociales digitales, actividades escolares, jóvenes universitarios, Tecnologías de Información y Comunicación.

\begin{abstract}
The main purpose of this work is to provide elements of analysis that allow us to know and understand the school activities carried out by young university students through the use of digital social networks. To do this, we analyzed the data collected through a questionnaire and semi-structured interviews to a group of young students of the Universidad Veracruzana, institutional education, belonging to different academic areas and to the Xalapa campus, whose ages range between 18 and 35 years. The results allowed us to show certain peculiarities regarding the use given to networks in school environments, including the type of social networks that predominate, roles in which they are immersed, subjects, as well as the forms of organization and interaction with their peers and teachers. It is considered that this study provides several lines to work in future research projects that will allow us to know young contemporary students going through the university in the era of the digital culture with the objective to contribute to the design of academic activities that promote the reflection on an improvement of the teaching learning process. This work was carried out within the framework of the Digital Literacy Thematic Network in the University, recognized and approved by the National Council of Science and Technology.
\end{abstract}

KEY WORDS: Digital social networks, school activities, university students, Information and Communication Technologies.

\footnotetext{
${ }^{1}$ Este artículo es producto de la Red Temática Literacidad Digital en la Universidad, reconocida y aprobada por el Consejo Nacional de Ciencia y Tecnología, desde mayo del 2016, conformada por investigadores, académicos y estudiantes de las siguientes instituciones: Universidad Veracruzana, Universidad de Sonora, Universidad Estatal de Sonora, Universidad Autónoma de Ciudad Juárez, Universidad Autónoma Benito Juárez de Oaxaca y el Centro de Investigación en Lengua Escrita y Matemáticas S.C.

2 Doctora en Pedagogía por la Universidad Nacional Autónoma de México. Investigadora del Programa de Investigación e Innovación en Educación Superior de la Universidad Veracruzana. Miembro de la Red Temática Literacidad Digital en la Universidad. Es miembro del Sistema Nacional de Investigadores de México, nivel 1. Correo: rociolopez@uv.mx

${ }^{3}$ Estudiante de la licenciatura en Psicología de la Universidad Veracruzana. Becaria y ayudante de Investigación en el Programa de Investigación e Innovación en Educación Superior de la Universidad Veracruzana. Su principal línea de investigación es: uso de las tecnologías digitales y las prácticas letradas digitales. Correo: pauletlp@gmail.com
}

Artículo recibido: 16 de febrero de 2017

Artículo enviado a corrección: 23 de marzo de 2017

Aprobado: 18 de mayo de 2017

Lópezy López doi: https://doi.org/10.36799/el.v2i1.45 Volumen 2, número 1, Año 2017, ISSN: 2448-5942 


\section{INTRODUCCIÓN}

En los últimos años, las redes sociales digitales ${ }^{4}$ se han convertido en recursos primordiales para los jóvenes universitarios, en las cuales realizan actividades de carácter personal, social y escolar; a través de ellas acceden a una gama de información, interactúan, socializan y se comunican, también comparten experiencias y expresiones de su realidad.

Actualmente en los pasillos universitarios es común escuchar frases como: “jte mando un Whats más tarde!” o “¿cómo estás en Face, para agregarte?”; incluso, cada vez es más frecuente escuchar a los docentes decir "hagan un grupo de Facebook para esta clase", o que los estudiantes realicen diversas actividades académicas, compartiendo información a través de foros de discusión, listas de correos, entre otros aspectos; no obstante, aún es escasa la investigación al respecto.

En la revisión de la literatura realizada por diversos autores (Ortiz 2012; Ortiz y López, 2013; López, 2014; Domínguez y López, 2015) sobre jóvenes universitarios y su relación tanto con las tecnologías digitales como con las redes sociales digitales se encontró que son escasos los trabajos en México que aborden el tema referente al acceso, uso y apropiación de las tecnologías digitales y, más aún, de las redes sociales entre las y los jóvenes universitarios, siendo a partir del 2011 cuando comienzan a difundirse, sobre todo en Internet.

Asimismo, se detectó una centralización de la investigación pues existe una mayor producción de conocimiento en universidades ubicadas en la Ciudad México, con estudiantes provenientes de instituciones públicas; principalmente dirigidas a conocer cómo Facebook y Twitter impactan la vida escolar, es decir, se enfocan en indagar si potencian o disminuyen el rendimiento escolar, dejando de lado, por una parte, otras redes sociales digitales importantes para el análisis y la reflexión como Twitter, YouTube, Whats App, ${ }^{5}$ entre otros aspectos relevantes que nos ayuden a conocer a los jóvenes estudiantes contemporáneos que transitan por la universidad, y al diseño de actividades académicas que contribuyan a su formación.

\footnotetext{
${ }^{4}$ A pesar de que no se ha realizado un consenso para definirlo, en el marco de este trabajo al hablar de redes sociales digitales, se hace referencia a la evolución que ha tenido internet, donde los usuarios pueden interactuar de forma activa y simultánea con otros usuarios generando una relación mediada por un aparato tecnológico (Crovi, López y López, 2009; Domínguez, López y Ortega, 2016).

${ }^{5}$ Cabe aclarar, que si bien, Whats $A p p$ es considerado como mensajería instantánea, incluso el correo electrónico, en este trabajo se catalogan como redes sociales digitales, considerando la voz de los estudiantes universitarios quienes indican que a través de éstas pueden socializar, entretenerse, recibir y compartir información, etcétera (Crovi, 2017).
}

Lópezy López doi: https://doi.org/10.36799/el.v2i1.45 Volumen 2, número 1, Año 2017, ISSN: 2448-5942 
Por otra parte, se identificó que existen estudios en México que se enfocan en proporcionar datos cuantificables sobre los hábitos que tienen los jóvenes con relación al uso de las tecnologías digitales (AMIPCI, 2011, 2015; INEGI, 2010, 2013; IAB, 2010) en los cuales se puede corroborar el creciente acceso y uso de las tecnologías en el transcurso de los años, así como, el impacto cultural, sobre todo en el sector juvenil (de 12 a 24 años de edad). También, los datos vislumbran, de cierta forma, el impacto que han tenido las tecnologías, específicamente la computadora e internet en el terreno educativo, así como, que el impacto y uso de las tecnologías -computadora e internet- se presenta principalmente en poblaciones de élite, en la cultura juvenil escolarizada.

Asimismo, los resultados muestran que los jóvenes mexicanos son quienes más se comunican a través de las redes sociales digitales, siendo éstas una de sus actividades favoritas. Envían mensajes privados y públicos; se comunican con amigos y familiares; dan seguimiento y opinan sobre cultura, deportes y entretenimiento; se enteran de las últimas noticias nacionales e internacionales, y lo hacen principalmente a través de Facebook y YouTube. Si bien, los datos son importantes para conocer el ambiente en el que se desenvuelven los jóvenes, éstos son generales y no permiten ver particularidades de carácter social y cultural ni mucho menos referentes al terreno educativo.

Los estudios, hasta el momento, nos indican los tipos de participación de los jóvenes estudiantes universitarios mexicanos en las redes sociales digitales (González, Becerra y Yánez, 2016), indican que éstas facilitan el aprendizaje ya que los jóvenes las utilizan en clase, elaboran trabajos y tareas y comparten información relacionada (Chávez y Gutiérrez, 2015). De igual forma, apuntan que no existe un patrón entre la capacidad de memorización de los estudiantes universitarios y las horas que estos invierten en Facebook; no se aprecia que el género, la edad o el área estén fuertemente relacionados con la capacidad de memorización (Valerio, Leyva, y Caraza y Rodríguez-Martínez, 2013). Tanto las mujeres como los hombres están usando las redes sociales para socializar, estar en contacto con su familia, conocer productos, buscar información sobre salud, religión y asuntos espirituales (Espinoza y Jiménez, 2013).

No obstante, hasta el momento no ha quedado claro cuáles son los usos que los jóvenes hacen de las redes sociales digitales. En las pocas investigaciones realizadas, no hay claridad acerca de los temas que les interesan, cómo es su interacción o expresión, sus formas de

Lópezy López doi: https://doi.org/10.36799/el.v2i1.45 Volumen 2, número 1, Año 2017, ISSN: 2448-5942 
organización, qué escriben, qué leen, qué aprendizajes adquieren los estudiantes universitarios a través del uso de las redes sociales digitales, y de los dispositivos en general; es decir, si de algún modo las redes sociales han contribuido en ampliar sus conocimientos (más allá del ámbito escolar), entre otros aspectos que contribuyan a formación profesional de los estudiantes.

Sin duda alguna, los jóvenes estudiantes están integrados activamente al mundo digital, cada vez son más quienes ingresan a la universidad sabiendo utilizar diversos y diferentes aparatos tecnológicos, con distintas lógicas de pensamiento, con diferentes formas de aprendizaje y elaboración de sus trabajos escolares, con otras formas de captar la realidad. Muchos de ellos forman parte de una generación que ha crecido inmersa en las nuevas tecnologías, convirtiéndose internet y otros aparatos o sistemas tecnológicos en parte integral de sus vidas y en su realidad tecnológica. Sus estilos de vida no son los mismos que los de las generaciones anteriores, ni siquiera es igual al de quienes vivieron una década atrás, porque su vinculación y su dominio sobre las TIC resulta cada vez mayor (Crovi, Garay, López y Portillo, 2011).

Es en este contexto que se desarrolló el proyecto PRODEP (Programa para el Desarrollo Profesional Docente), titulado "Uso de las redes sociales virtuales entre los estudiantes universitarios" (UV-PTC-763), el cual tuvo como propósito generar información acerca del uso que les dan los jóvenes estudiantes de la Universidad Veracruzana a las redes sociales digitales, tanto en el ámbito social como en el escolar. Se trabajó con los estudiantes de licenciatura, de 18 a 35 años, del sistema escolarizado, campus Xalapa, distribuidos en seis de las áreas académicas de la UV: Artes, Biológico-Agropecuaria, Ciencias de la Salud, Económico-Administrativa, Humanidades y Técnica. ${ }^{6}$ Particularmente, este trabajo se centra en el análisis referente al uso de las redes sociales digitales en el ámbito escolar. ${ }^{7}$ Para la obtención de información se definieron dos etapas; a continuación, se describe cada una de ellas.

\section{MÉTODO}

${ }^{6} \mathrm{La}$ UV cuenta con una presencia en 28 municipios a lo largo del territorio veracruzano, dentro de cinco grandes regiones: Xalapa, Veracruz, Córdoba-Orizaba, Poza Rica-Tuxpan, Coatzacoalcos-Minatitlán.

${ }^{7}$ Este artículo es producto de la Red Temática Literacidad Digital en la Universidad (RED-LDU), reconocida y aprobada por CONACyT desde mayo del 2016, conformado por investigadores, académicos y estudiantes de diversas instituciones de educación superior nacionales y del extranjero.

Lópery López doi: https://doi.org/10.36799/el.v2i1.45 Volumen 2, número 1, Año 2017, ISSN: 2448-5942 
En una primera etapa se aplicó un cuestionario a un total de 594 estudiantes pertenecientes a diversos programas educativos ${ }^{8}$ (Véase Tabla 1). Los estudiantes procedían en su mayoría del estado de Veracruz, pero también se registraron de otras entidades cercanas como Puebla, Tabasco, Tamaulipas y Oaxaca (55\% de mujeres y $45 \%$ de hombres).

Dicho cuestionario se estructuró en seis secciones: 1) Datos personales (género, edad, trabajo y actitud al navegar en Internet); 2) Cultura Digital (percepciones en los cambios en dispositivos, navegación en Internet y habilidades digitales); 3) Participación en las redes (en el que se plantearon diversas temáticas como medio ambiente, educación, trabajo, arte, entretenimiento, sociedad, derechos humanos, política y religión); 4) Niveles de interacción (frecuencia, habilidades y plataformas, así como temáticas detonadoras), 5) Actividades escolares (redes que utilizan, acciones realizadas, participación en grupos y opiniones) y 6) nivel socioeconómico.

Tabla 1. Programas y número de estudiantes participantes

\begin{tabular}{|c|c|c|}
\hline Área de conocimiento & Programas & Muestra \\
\hline \multirow{2}{*}{ Artes } & Música & 37 \\
\cline { 2 - 3 } & Fotografía & 5 \\
\hline \multirow{2}{*}{ Bilógico-Agropecuaria } & Ingeniero Agrónomo & 17 \\
\cline { 2 - 3 } & Biología & 17 \\
\hline
\end{tabular}

\footnotetext{
${ }^{8}$ Se buscó un número óptimo de representatividad para este campus, incluyendo los programas de mayor y menor matrícula dentro de las seis áreas de conocimiento, considerando el período escolar 2013-2014 (UV, 2015).

${ }^{9}$ Se elaboró en el marco del proyecto Jóvenes y cultura digital. Nuevos escenarios de interacción social. Fue aplicado en la Universidad Nacional Autónoma de México, Universidad Pedagógica Nacional del Ajusco, Universidad Autónoma Metropolitana-unidad Lerma, Universidad de Sonora y Universidad Veracruzana (Crovi y Lemus, 2014).
}

Lópery López doi: https://doi.org/10.36799/el.v2i1.45 Volumen 2, número 1, Año 2017, ISSN: 2448-5942 


\begin{tabular}{|c|c|c|}
\hline \multirow{2}{*}{ Ciencias de la Salud $^{10}$} & Enfermería & 45 \\
\cline { 2 - 3 } & Médico Cirujano & 39 \\
\hline \multirow{2}{*}{ Económico-Administrativa } & Administración & 138 \\
\cline { 2 - 3 } Humanidades & Geografía & 32 \\
\hline \multirow{2}{*}{ Técnica } & $\begin{array}{c}\text { Antropología } \\
\text { Lingǘstica }\end{array}$ & 137 \\
\hline \multirow{2}{*}{ Derecho } & 103 \\
\cline { 2 - 3 } & Arquitectura & 15 \\
\hline \multicolumn{2}{|c|}{ Total } & 594 \\
\hline
\end{tabular}

Los resultados obtenidos en esta etapa de la investigación (que culminó en junio de 2015), fueron la base sustancial que permitió conocer los rasgos que los estudiantes comparten con otros jóvenes de su generación: el comportamiento que manifestaron tener al utilizar las redes sociales digitales; redes sociales que más utilizan, temas de interés, espacios (físicos o digitales) donde participan; nivel de interacción en redes sociales, así como, el uso de estas herramientas en el ámbito escolar. ${ }^{11}$

Debido al interés de profundizar en diversos hallazgos detectados en esa fase cuantitativa, se optó por investigar la parte cualitativa, por lo que se realizaron diversas entrevistas semiestructuradas a estudiantes que habían respondido el cuestionario y que, por lo tanto, estaban familiarizados con el tema de la investigación. La estructura de la entrevista constó de cuatro apartados: a) Vida Cotidiana (Social y Escolar); b) Acceso y Uso de los Dispositivos Digitales; c) Redes Sociales Digitales; y d) Activismo Social en la Red.

Para la selección de los participantes se consideraron los diversos tipos de comportamiento que los estudiantes indicaron tener, en el cuestionario, al navegar en las redes. Las actitudes más sobresalientes en la primera etapa fue la de Enlace (tienen interacción con

\footnotetext{
${ }^{10}$ Por ser parte de un proyecto coordinado con otras universidades mexicanas, las cuales compartimos los mismos instrumentos metodológicos, el estudio de la UV en el caso del área de Ciencias de la Salud, tuvo que descartar la carrera de Psicología, que es la de mayor matrícula, reemplazándola por la carrera de Medicina. Ello se debió a que otros programas académicos ubican a Psicología en un área de conocimiento diferente.

11 Para conocer más sobre los resultados del cuestionario y otros aspectos de la investigación, véase: López, Domínguez y Quiñones (2015); López y Anaya (2016); López y Domínguez (2016); Crovi (2017).
}

Lópery López doi: https://doi.org/10.36799/el.v2i1.45 Volumen 2, número 1, Año 2017, ISSN: 2448-5942 
algunas personas, les agrada hacerlo, pero se comunican sólo cuando hay razones importantes) con un 67\%, seguida de la Sociable (tienen interacción con muchas y diversas personas constantemente) con un 16\% y Selectivo (tienen interacción con pocas personas, pero consideran que no tienen muchas razones para comunicarse o no les gusta hacerlo) con un $14 \% .^{12}$

En total se realizaron 20 entrevistas: 9 casos de jóvenes que indicaron tener una actitud de Enlace, 5 una actitud Sociable, 5 una actitud Selectiva y 1 que indicó no sentirse identificado con Ninguno de los tipos de comportamientos ofrecidos (Véase Tabla 2). Cabe destacar que, dentro de estos casos, se buscó un equilibro tanto del tipo de comportamiento al navegar como en aspectos de género.

Tabla 2. Datos generales de los jóvenes entrevistados en la UV

\begin{tabular}{|c|c|c|c|}
\hline Tipo de comportamiento & Área académica & Edad & Entrevistado \\
\hline \multirow{3}{*}{ Técnica } & 20 & Estudiante 1 \\
\cline { 3 - 4 } & & 19 & Estudiante 2 \\
\cline { 3 - 4 } & & 19 & Estudiante 3 \\
\cline { 2 - 4 } & Económico- & 19 & Estudiante 4 \\
\hline
\end{tabular}

${ }^{12}$ En el cuestionario existía la posibilidad de elegir otras dos opciones: "Discreto" o "Ninguno"; sin embargo, solo un $1 \%$ se identificó con la primera y un $2 \%$ con la segunda (solo uno de éstos jóvenes accedió al proceso de entrevista).

Lópery López doi: https://doi.org/10.36799/el.v2i1.45 Volumen 2, número 1, Año 2017, ISSN: 2448-5942 


\begin{tabular}{|c|c|c|c|}
\hline \multirow[t]{5}{*}{ Enlace } & Administrativa & 20 & Estudiante 5 \\
\hline & \multirow{2}{*}{$\begin{array}{c}\text { Biológico- } \\
\text { Agropecuaria }\end{array}$} & 21 & Estudiante 6 \\
\hline & & 20 & Estudiante 7 \\
\hline & Artes & 22 & Estudiante 8 \\
\hline & Ciencias de la Salud & 20 & Estudiante 9 \\
\hline \multirow{5}{*}{ Selectivo } & \multirow{2}{*}{$\begin{array}{c}\text { Biológico- } \\
\text { Agropecuaria }\end{array}$} & 20 & Estudiante 10 \\
\hline & & 19 & Estudiante 11 \\
\hline & \multirow{2}{*}{ Ciencias de la Salud } & 21 & Estudiante 12 \\
\hline & & 20 & Estudiante 13 \\
\hline & $\begin{array}{l}\text { Económico- } \\
\text { Administrativa }\end{array}$ & 20 & Estudiante 14 \\
\hline \multirow{5}{*}{ Sociable } & \multirow{2}{*}{ Humanidades } & 21 & Estudiante 15 \\
\hline & & 19 & Estudiante 16 \\
\hline & Ciencias de la Salud & 21 & Estudiante 17 \\
\hline & Artes & 25 & Estudiante 18 \\
\hline & Geografía & 21 & Estudiante 19 \\
\hline Ninguno & Humanidades & 20 & Estudiante 20 \\
\hline
\end{tabular}

Las entrevistas fueron realizadas entre agosto y diciembre de 2015. Cabe aclarar que el número final de participantes dependió de la accesibilidad y disponibilidad de los jóvenes, ya que si bien se identificaron jóvenes que seleccionaron un tipo de comportamiento, al momento de la búsqueda para realizar las entrevistas fue complejo localizarlos, principalmente porque no 
compartieron datos personales (matrícula o correo para contactarlos), tal como fue el caso de los discretos.

Para la realización de este trabajo se recuperaron diversos elementos, de ambas etapas, particularmente, el uso que le dan los estudiantes a las redes sociales digitales en el ámbito escolar.

\section{RESULTADOS}

A continuación, se presentan los resultados, considerando tres aspectos: 1) Redes sociales digitales: significado, cambios y redes más utilizadas; 2) Dinámicas de organización y roles de los estudiantes en las redes sociales digitales; 3) Opiniones de los jóvenes con relación al uso de las redes en el ámbito escolar y el papel de las disciplinas.

\section{Redes sociales digitales: significado, cambios y redes más utilizadas}

Hay que reconocer que la vida de los jóvenes universitarios se encuentra llena de diversas actividades, donde existen algunas que no logran identificarse a simple vista, pero se sabe que están inmersas en sus vidas, como es el caso de las redes sociales digitales. En este sentido, en la primera parte de las entrevistas se indagó sobre la vida cotidiana de los estudiantes y las diversas actividades que realizan, con el fin de identificar momentos donde éstos acceden a las redes sociales digitales, y a los recursos digitales en general.

En un primer momento, no apareció en el discurso de los jóvenes el tema referente al uso de las redes sociales digitales (y de las TIC en general), sin embargo, a medida que se profundizó en las entrevistas, se dejó entrever que las utilizan en diversos ámbitos y momentos. Algunos jóvenes hicieron hincapié en diversas actividades que antes eran realizadas de manera física o verbal y que se han traslado particularmente a las redes sociales digitales, por ejemplo, la comunicación entre estudiantes y docentes, tal como lo indicó un estudiante:

La red social facilita enviar y recibir documentos, formatos, archivos de cualquier tipo, entonces es una manera más: 'más' práctica de estar en contacto con tu equipo en este caso si haces un trabajo en equipo, también hasta con los maestros los maestros también... Dime quién no tiene Facebook, ¿no? [Sí, claro] En este caso y ellos también están ahí, este, al tanto de lo que sucede. A veces, este... tengo muchos

Lópery López doi: https://doi.org/10.36799/el.v2i1.45 Volumen 2, número 1, Año 2017, ISSN: 2448-5942 
maestros que en realidad sí toman en serio lo que es la red social (Estudiante 20, 20 años, estudiante de Derecho).

Al preguntar a los jóvenes en las entrevistas qué entienden por redes sociales digitales, éstos los relacionaron con palabras como: comunicación, espacios, compartir, interacción, vivencias y des-estrés. En donde la palabra “comunicación” es una de las más mencionadas; sin embargo, para los jóvenes va más allá de compartir un simple mensaje, ya que reconocieron que gracias a ellas pueden tener una comunicación asincrónica, pues no necesitan estar frente a la persona para poder comunicarse.

A su vez, reconocieron que gracias al uso de las redes sociales pueden conocer gente e incrementar su círculo social, por lo que además las consideran como las "nuevas formas de relación personal". Otros estudiantes las describieron como un espacio en donde pueden realizar múltiples actividades, tal como se expresa en los siguientes testimonios: "cualquier espacio donde yo pueda interactuar con otras personas en el que no necesariamente tenga que conocerlas de forma física” (Estudiante 1, 20 años, estudiante de Ingeniería en Alimentos); "Es un vínculo, una señal que conecta con varios usuarios a la misma vez para poder estar interactuando ¿no? en el mismo tiempo pero no en el mismo lugar" (Estudiante 6, 21 años, estudiante de Agronomía); "Para mí una red social es un lugar en la web [...] dándole un uso múltiple, no necesariamente para distraerme [...] puedo estar en un mismo chat comunicándome con alguna persona $[\ldots]$ y en otro chat puedo estar poniendo algún trabajo, enviando algún documento... (Estudiante 17, 21 años, estudiante de Medicina).

En general, los jóvenes reconocieron que a partir del uso de las redes sociales digitales han sufrido transformaciones: cambios tanto "positivos" como "negativos". Por un lado, mencionaron que facilitan la comunicación, la recepción y el envío de información, divulgación de contenidos, incluso hacen mención de los costos, los cuales son más baratos si son comparados con las tarifas de años anteriores, etcétera. Por otro lado, además de la pérdida de tiempo, comentaron que han dejado de hacer actividades que antes solían hacer cotidianamente, como leer o tratar a las personas cara a cara, ahora enfocan su atención en las redes sociales para ver "qué es lo que hay".

Lópery López doi: https://doi.org/10.36799/el.v2i1.45 Volumen 2, número 1, Año 2017, ISSN: 2448-5942 
Ahora bien, al preguntarle a los jóvenes, en el cuestionario, cuáles eran las redes sociales digitales y recursos digitales que más utilizan, éstas fueron Facebook (64\%) y Whatsapp (36\%). En menor medida aparecieron otros tipos de redes sociales digitales, tales como: YouTube; correo electrónico, Instagram, Twitter, Pinterest, Tumblr y Snapchat. De acuerdo con las entrevistas, a través de Facebook y Whatsapp, los estudiantes realizan determinadas actividades: "Facebook para subir fotos o compartir videos o a veces tareas; hacemos grupos para enviarnos tareas o pasarnos cosas; para comunicarme con amigos que a veces no tengo en Whatsapp o algo así" (Estudiante 4, 19 años, estudiante de Administración); “Facebook, o mejor dicho el Messenger de Facebook, lo ocupo para hablar con las personas con las que no puedo hablar por el Whatsapp, y Whatsapp la ocupo por lo regular para hacer muchos grupos y tener nociones distintas de ello" (Estudiante 1, 20 años, estudiante de Ingeniería en Alimentos); “Facebook obviamente para socializar más ahí, y ya Whatsapp que es algo muy personal, relación con personas más cercanas o familiares" (Estudiante 15, 21 años, estudiante de Derecho).

$\mathrm{Al}$ indagar sobre los temas de interés en el cuestionario, se identificó que a un 27\% le interesan los temas educativos/académicos; su mayor interés se concentra en temas relacionados con el ocio, diversión y entretenimiento (29\%). Por ejemplo, un porcentaje considerable $(25 \%)$ indicó interesarse por temas culturales o artísticos. Los temas menos abordados fueron los religiosos (39\%) y políticos (34\%), ya que son los que respondieron en estar nada comprometidos con éstos temas.

A pesar de que la mayoría de los estudiantes coincide en privilegiar los temas de entretenimiento, en las entrevistas también aparecieron aspectos referentes a la ciencia, deportes, ambientales, problemas sociales y a las relaciones de pareja, tal y como se muestra en los siguientes comentarios: "Pues de todo tipo de deportes e informativa; a veces de ciencias..."

(Estudiante 14, 20 años, estudiante de Administración); "En Whatsapp el novio ¿no? amor, a veces tareas que la exposición de mañana, o platicar con los amigos etc.” (Estudiante 12, 21 años, carrera de Enfermería); “Temas a veces de los animales, estoy muy interesado en la protección hacia los animales..." (Estudiante 4, 19 años, estudiante de Administración); "Por lo regular abordo temas educativos, temas científicos o académicos...” (Estudiante 6, 21 años, estudiante de Agronomía).

Lópery López doi: https://doi.org/10.36799/el.v2i1.45 Volumen 2, número 1, Año 2017, ISSN: 2448-5942 
Por otra parte, respecto a las acciones que señalaron los estudiantes (en el cuestionario) al utilizar las redes sociales digitales, éstas se concentraron en visualizar y descargar; no obstante, como se puede observar en la Tabla 3, las acciones varían dependiendo de la actividad que quieren realizar y su finalidad.

Tabla 3. Acciones de los estudiantes en las redes sociales digitales ( $\%)$

\begin{tabular}{|c|c|c|c|c|c|c|}
\hline Acciones & \multicolumn{2}{|c|}{$\begin{array}{c}\text { Textos, libros, } \\
\text { artículos }\end{array}$} & \multicolumn{2}{c|}{$\begin{array}{c}\text { Material audio-visual } \\
\text { (videos, imágenes, } \\
\text { etcétera) }\end{array}$} & \multicolumn{2}{|c|}{$\begin{array}{c}\text { Información } \\
\text { académica, eventos, } \\
\text { convocatorias }\end{array}$} \\
\hline & Sí & No & Sí & No & Sí & No \\
\hline Visualizar & 69 & 31 & 77 & 23 & 74 & 26 \\
\hline Dar like & 24 & 76 & 43 & 57 & 35 & 65 \\
\hline Compartir & 42 & 58 & 61 & 39 & 53 & 47 \\
\hline Comentar & 27 & 73 & 43 & 57 & 43 & 57 \\
\hline Descargar & 68 & 32 & 68 & 32 & 46 & 54 \\
\hline $\begin{array}{c}\text { Enviar (archivos, } \\
\text { mensajes, etc.) }\end{array}$ & 46 & 54 & 58 & 42 & 50 & 50 \\
\hline Conversar & 24 & 76 & 35 & 65 & 38 & 62 \\
\hline Publicar & 28 & 72 & 42 & 58 & 37 & 63 \\
\hline
\end{tabular}

De igual forma, durante las entrevistas, se pudo observar que las acciones que más realizan los jóvenes en sus redes sociales digitales se enfocan en ver, compartir, dar like y consumir contenido. En muy pocos casos se busca algún tipo de debate o retroalimentación por parte de los amigos o del público en general. Algunas de las expresiones fueron las siguientes: "Yo casi no publico, soy pasiva en cuanto a publicaciones, pero sí soy de las personas que dan likes..." (Estudiante 2, 19 años, estudiante de Ingeniería en Alimentos); “Digamos que me gusta más como... checar todo lo que comparten las demás personas y comentar al respecto [...] no me gusta tanto publicar cosas serias porque siento que no es el espacio para darle la importancia que deberían tener (Estudiante 1, 20 años, estudiante de Ingeniería en Alimentos); "Yo casi no publico, muy de vez en cuando, no suelo ser de esos, yo ocupo la red social para lo que se creó 
[...] para estar en comunicación con otras personas...” (Estudiante 20, 20 años, estudiante de Derecho).

\section{Dinámicas de organización y roles de los estudiantes en las redes sociales digitales}

Dentro del tema de redes sociales fue posible encontrar varias similitudes por carrera, según el uso que los estudiantes les dan a éstas, una de las coincidencias es que todas y todos los entrevistados han participado por lo menos una vez en un grupo escolar creado en Facebook o Whatsapp, a su vez indicaron que el uso más común en estas redes es para enviar y recibir archivos, así como dar avisos.

De acuerdo con los datos del cuestionario, un 86\% de los estudiantes señaló que participó en un grupo dentro de alguna experiencia educativa (materia) dentro de una red social; el 14\% restante indicó que no lo hace. Al averiguar quién crea comúnmente los grupos en los que participan, la mayoría comentó en el cuestionario que fue un compañero (77\%); solo 6\% señaló que el grupo lo crearon ellos mismos; solo un 17\% indicó que el grupo lo crearon los profesores.

Un 86\%, respondió que sí se comunica con sus profesores a través de las redes, básicamente para enviar tareas $(84 \%)$ y para resolver dudas de la clase $(75 \%)$, solo un grupo reducido de jóvenes indicó que tienen tutorías académicas (34\%). Esto también se reflejó en las entrevistas:

Por lo regular tenemos grupos, por ejemplo, de educación quirúrgica, tenemos el grupo de mi salón, el de mi generación, tenemos el grupo de la facultad de medicina, tengo mi grupo de amigos (Estudiante 17, 21 años, estudiante de Medicina).

En Facebook compartimos archivos de clases que los maestros nos dan y los compartimos entre todos para que todos los tengamos y pues ya depende de ti si lo imprimes, lo tienes en tu celular o lo ocupas digitalmente (Estudiante 7, 20 años, estudiante de Agronomía).

Por lo regular tenemos un grupo con el propio maestro. Si tenemos alguna duda, necesitamos saber dónde está o si alguien lo ha visto mandamos un WhatsApp y Facebook; es para subir o descargar documentos, compartirles trabajos a los 
compañeros; incluso si vamos a hacer un trabajo en equipo, yo hago mi parte y otros hacen su parte, pero cada quien en su casa y sólo lo subimos a Facebook (Estudiante 13, 20 años, Ciencias de la Salud).

Lo utilizamos para ponernos de acuerdo para ensayos o decir, esta pieza vamos a verla hoy o mañana, hay que practicarla, lleven su partitura (Estudiante 8, 22 años, estudiante de Música).

Un 14\% restante (los que no se comunican con sus profesores), señalaron que no lo hacen por los siguientes motivos: "la mayoría de los profesores no utiliza redes sociales"; "veo a mis profesores en la escuela"; "no tengo agregados a mis profesores, para tener libertad de expresión"; "no me interesa agregar a mis profesores", es decir, existe un grupo de estudiantes a los que no les interesa tener comunicación con sus profesores por estas vías digitales.

Cabe enfatizar que en las entrevistas se pudo observar que los roles juegan un papel relevante en el uso de las redes, como es el caso de los jefes de grupo, pues son ellos quienes tienen el compromiso de mantener informada a la comunidad estudiantil sobre eventos académicos y diversas acciones sociales y escolares; generalmente son quienes establecen contacto directo con el maestro y, a su vez, mantienen actualizados los grupos creados en las redes, como fue el caso de un estudiante, quien expresó lo siguiente:

Soy jefe de grupo, entonces estoy en contacto con varios doctores y por lo regular avisan: 'Carlos, avísale a tu grupo que no vamos a poder tener clase porque aún estoy en cirugía'; cualquier aviso que me den lo publico en un grupo en Whats $A p p$ donde estamos todos mis compañeros (Estudiante 17, 21 años, Ciencias de la Salud).

De igual forma son los representantes de generación, como lo indicó Rodrigo: "Pues me volví representante de generación y a partir de eso yo empecé a querer informarme más sobre las cosas, ¿no?, y también actos que no tienen que ver con lo universitario, de gente con la que yo he estado que me ha influenciado mucho en mi vida para involucrarme o interesarme por ese tipo de cosas, o cosas que he leído y así” (Estudiante 18, 25 años, Artes).

Lópery López doi: https://doi.org/10.36799/el.v2i1.45 Volumen 2, número 1, Año 2017, ISSN: 2448-5942 


\section{Opiniones de los jóvenes con relación al uso de las redes en el ámbito escolar y el papel de las disciplinas}

La mayoría de los jóvenes reconocieron en las redes sociales digitales una herramienta de gran utilidad en el espacio escolar, más de la mitad de la población juvenil está de acuerdo con diversas afirmaciones: les permiten realizar sus tareas en equipo en cualquier tiempo y espacio; mejoran la comunicación con sus compañeros; les permiten hacer tareas más creativas y dinámicas; les ahorran tiempo para hacer sus trabajos escolares (Véase Tabla 4). No obstante, llama la atención que prácticamente en todos los enunciados indicados hay una tercera parte de la población estudiantil que se mostró indecisa sobre lo que se les plantea.

Tabla 4. Opiniones de los jóvenes con relación al uso de las redes sociales digitales (\%)

\begin{tabular}{|c|c|c|c|c|c|}
\hline Afirmaciones & 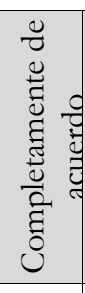 & $\begin{array}{l}\text { o } \\
\bar{\Xi} \\
\tilde{U} \\
\tilde{E} \\
\tilde{D}\end{array}$ & 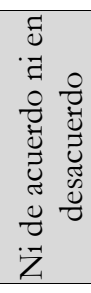 & 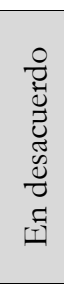 & 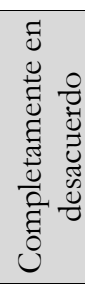 \\
\hline Todos los profesores deben utilizarlas para enseñar & 13 & 31 & 44 & 10 & 2 \\
\hline $\begin{array}{c}\text { Me permiten realizar trabajos en equipo en cualquier } \\
\text { tiempo y espacio }\end{array}$ & 33 & 51 & 12 & 3 & 1 \\
\hline Me saturan de información & 13 & 30 & 41 & 15 & 1 \\
\hline Me permiten realizar tareas más creativas y dinámicas & 29 & 45 & 21 & 4 & 1 \\
\hline $\begin{array}{l}\text { Mejoran mi comunicación e interacción con mis } \\
\text { compañeros }\end{array}$ & 27 & 36 & 28 & 7 & 2 \\
\hline Ahorran tiempo para preparar trabajos escolares & 30 & 38 & 24 & 7 & 1 \\
\hline $\begin{array}{c}\text { Todos mis compañeros deben utilizarlas para realizar } \\
\text { actividades académicas }\end{array}$ & 16 & 25 & 46 & 11 & 2 \\
\hline No encuentro rápidamente información deseada & 5 & 14 & 38 & 37 & 6 \\
\hline Fomentan el trabajo en grupo y colaborativo & 14 & 28 & 41 & 15 & 4 \\
\hline Me distraen para realizar mis trabajos escolares & 18 & 34 & 32 & 14 & 2 \\
\hline $\begin{array}{l}\text { Prefiero realizar mis actividades académicas en ellas que } \\
\text { en una plataforma institucional }\end{array}$ & 20 & 24 & 35 & 15 & 6 \\
\hline
\end{tabular}

Lópery López doi: https://doi.org/10.36799/el.v2i1.45 Volumen 2, número 1, Año 2017, ISSN: 2448-5942 
Un grupo importante de jóvenes indicó que las redes sociales digitales tienen opiniones no muy favorables, tales como: saturación de información y dificultad de identificar sitios confiables; dificultad para encontrar rápidamente la información deseada y son un distractor a la hora de hacer trabajos escolares. Solo una tercera parte de la población juvenil coincidió en que todos los profesores deberían utilizarlas para enseñar, el resto se mostraron indecisos, lo que invita a reflexionar sobre las ventajas que dice tener el uso de éstas en el proceso de enseñanzaaprendizaje, más aún en las plataformas institucionales.

Como se puede observar en la Tabla 4, la mayoría de las y los estudiantes comentaron que prefieren realizar sus actividades académicas en las redes sociales digitales en lugar de Eminus (plataforma institucional de la UV), tal vez porque suelen ser más amigables y prácticas, pero, sobre todo, porque no es un espacio en el que se manifieste una pertenencia institucional y esto les concede más libertad.

Uno de los datos relevantes que se identificó en las entrevistas fue que, indiscutiblemente, las disciplinas académicas jugaron un papel importante en el uso que les dan los jóvenes a las redes sociales digitales, es decir, de acuerdo a la formación académica de los estudiantes, será el uso que tengan las redes, por ejemplo, los estudiantes de Derecho tratan temas relacionados con los derechos humanos; los estudiantes de Medicina y Enfermería comparten información referente a enfermedades y avances médicos; los estudiantes de Ingeniería en Alimentos plantearon temas referentes a los transgénicos.

En general, los estudiantes indicaron que a través de las redes sociales digitales pueden mantenerse actualizados en cuestiones de innovación relacionadas a sus carreras, pues con la ayuda de estas se mantienen en contacto directo con diversas personas que tratan temas relacionados a sus intereses, por lo que no sólo están en contacto con la persona sino también con sus trabajos académicos y eso les ayuda a poder consultar y compartir información con gente especializada.

Algunas de sus expresiones fueron las siguientes: "Como estudiamos geografía luego platicamos sobre temas del ámbito social, problemáticas, problemas políticos, sociales; en eso es en lo que nos basamos más que nada nosotros" (Estudiante 19, 21 años, EconómicoAdministrativa); "Hay varias páginas que abordan temas de biología, entonces luego vienen links

Lópery López doi: https://doi.org/10.36799/el.v2i1.45 Volumen 2, número 1, Año 2017, ISSN: 2448-5942 
de artículos y los puedes encontrar más fácilmente” (Estudiante 11, 19 años, BiológicoAgropecuaria); "La arquitectura cambia día con día y cada vez hay más tecnología [...], entonces, pues tienes que estar leyendo, viendo y buscando información constantemente [...]; en Instagram y en Twitter puedes seguir a arquitectos famosos y ellos ponen su información o comparten cosas; ya es como un trato más directo con el arquitecto" (Estudiante 3, 19 años, Técnica); "Muchas personas abren paginas importantes en Facebook de medicina y de otra información; he encontrado muchos videos del sistema óseo y circulatorio [...], he encontrado hasta la filosofía de enfermería [...]. Sí he encontrado muchas cosas que ayudan a la carrera y he aprendido cosas que no sabía o no me quedaban claras” (Estudiante 13, 20 años, Ciencias de la Salud).

\section{DISCUSIÓN}

Los resultados de esta investigación permitieron dar a conocer el uso que los jóvenes estudiantes de la Universidad Veracruzana dan a las redes sociales digitales en el ámbito escolar. En general, se puede decir que las y los estudiantes presentaron varias similitudes con relación al uso de las redes sociales, pues reconocieron la importancia de éstas como fuentes de información alternativa o complementaria; también, mencionaron que en ellas se aloja información que es de utilidad en su formación profesional, y de fácil acceso a través de algunos links o clicks. Además, les permite mantenerse actualizados en temas relacionados a sus carreras.

Cabe destacar que la mayoría de los jóvenes utilizan preferentemente la red social de Facebook, en la cual reciben y envían documentos escolares (tareas y avisos), siendo el Smartphone el dispositivo más utilizado; mencionaron al menos seguir una página en esta red relacionada con su carrera, la cual consultan y comparten. En este sentido, sería enriquecedor seguir profundizando en el uso que se le da a esta red: ¿qué tipo de información etiquetan o comentan constantemente?, ¿Qué escriben? ¿Qué leen? ¿Qué significado les otorgan las y los estudiantes a las prácticas de lectura y escritura? ¿Quiénes los acompañan en este tipo de prácticas digitales? ¿Cómo aprendieron a utilizar este espacio? ¿Escriben más los estudiantes?, ¿Qué tipo de usos académicos le dan a Facebook?, entre otros aspectos.

Sin duda alguna, Facebook se ha convertido en una necesidad más allá de la comunicación y el entretenimiento, pues compartir vivencias cotidianas ha pasado a constituirse en una 
necesidad, siendo un espacio importante en su formación profesional, social y humana. A través de este recurso comparten lo que más les interesa, sus opiniones, pensamientos y emociones, además de que envían y reciben fotos y videos de experiencias personales o ajenas, así como de aspectos significantes que giran alrededor de sus vidas, por lo que sería un tema en el que se podría seguir profundizando.

No obstante, es importante reflexionar sobre el uso de dicha red en el ámbito académico, ya que, como lo indican (Túñez y Sixto, 2012, p. 81), si bien Facebook tiene ciertas ventajas (conversaciones privadas, respuestas inmediatas, información de convocatorias de actos o situaciones concretos, etcétera) entre las principales dificultades está el dar seguimiento de las actividades de los estudiantes de manera personalizada, así como, el impedimento de utilizar documentos en PDF, los cuales se tienen que convertir y publicar como imagen, lo que dificulta su lectura y las restricciones de visualización y descarga en algunas instituciones.

Sin bien, la mayoría indicó que se comunica con sus profesores a través de estas herramientas, un dato que resulta revelador es que la mayoría de los grupos no son creados por sus profesores, lo cual podría ser un reflejo de la falta de familiaridad, o interés, que tienen los profesores sobre el uso de las redes sociales digitales para potencializar los procesos de comunicación e interacción en el nivel universitario; de tal forma, no es extraño observar que las actividades que más realizan los estudiantes sean chatear y dar "me gusta", si sus profesores no promueven o no impulsan el vínculo entre los procesos educativos y las redes sociales con fines escolares y académicos.

El reto está, por una parte, en que los estudiantes tengan una participación más activa dentro de las redes en diversas temáticas, tanto las relacionadas con el ámbito académico, como las de carácter social-cultural, que contribuyan a su formación profesional y humana. Como bien lo indican Sixto, Aguado y Riveiro (2017, p. 49), "ya no basta con estar en la web, sino que hay que saber estar [...] implica una participación activa, colaborativa y solidaria con respecto a la comunidad".

Asimismo, es esencial que los estudiantes exploten los recursos que tienen a su alcance, por lo que hay que enseñar a los jóvenes universitarios métodos de búsqueda y discernir entre lo que sirve, y lo que no. En este sentido, los profesores juegan un papel fundamental para 
promover entre los estudiantes mayores habilidades y capacidades para la búsqueda de contenidos, nutrir su conocimiento y formación. Incluso, sería pertinente conocer e identificar el uso que le dan los docentes a las redes sociales digitales, y dispositivos en general, conocer qué utilidad le dan y sobretodo el aprovechamiento que le dan en el ámbito educativo.

En suma, los datos recabados permiten tener una primera radiografía referente al uso de las redes sociales digitales en el ámbito escolar, en este caso, un grupo de jóvenes de la UV. Es importante seguir profundizando sobre el tema, pues la información no es estática, es decir, evoluciona no sólo en la medida en que se renuevan los recursos digitales, sino también en la medida en que su impacto transforma las prácticas sociales y profesionales de los estudiantes universitarios.

Consideramos que la realización de estudios sobre estos temas no sólo permitirá conocer a los principales actores sociales que transitan día con día en los espacios universitarios, sino también podrá servir de base empírica para diseñar y orientar acciones que den respuesta a las necesidades, intereses y prioridades reales manifestadas por la comunidad estudiantil.

\section{REFERENCIAS}

Crovi, Delia; María de los Ángeles, López y Rocío, López. "Redes sociales: análisis y aplicaciones". México: Plaza Valdés, 2009.

Crovi, Delia. y Carlos, Lemus. "Jóvenes estudiantes y cultura digital: una investigación en proceso. Bitácora de la propuesta metodológica cuantitativa". [Versión electrónica] Virtualis, 5 (2014): 1-8.

Crovi, Delia. "Redes sociales digitales. Lugar de encuentro, expresión y organización para los jóvenes”. México: UNAM, 2017.

"Consumo de Medios Digitales en México". Interactive Advertising Bureau, IAB. 2010. 17 de diciembre 2016. http://www.amai.org/datos_files/BGJNY3.pdf

Domínguez, Fernando y Rocío, López. "Uso de las redes sociales digitales entre los jóvenes universitarios en México. Hacia la construcción de un estado del conocimiento". [Versión electrónica] Revista de comunicación, 14 (2015): 48-69.

Lópery López doi: https://doi.org/10.36799/el.v2i1.45 Volumen 2, número 1, Año 2017, ISSN: 2448-5942 
Domínguez, Fernando; Rocío, López y Juan Carlos, Ortega. "Hagan un grupo de Facebook para esta clase. ¿De qué hablamos cuando hablamos de redes sociales?” [Versión electrónica] Revista Interamericana de Educación de Adultos, 2 (2016): 117-126.

Espinoza, Elvia y Jiménez, Salvador. "Una mirada a las redes sociales virtuales desde el género" Gestión y estrategia, 43 (2013): 33-50.

"Estadísticas sobre disponibilidad y uso de tecnología de información y comunicación en los hogares 2009”. Instituto Nacional de Estadística Geografía e Informática-INEGI. 2010. 19 de diciembre 2016. http://www.inegi.org.mx.

González, Guadalupe, Becerra, Teresa y Yánez, Mireya. “Ciberactivismo: nuevas formas de participación para estudiantes universitarios”. [Versión electrónica] Comunicar. Revista de comunicación y educación, 46 (2016): 47-54.

Guzmán, Carlota y Claudia, Saucedo. "La investigación sobre alumnos en México: recuento de una década (1992-2002)". En Ducoing, Patricia (Coord.), Sujetos, actores y procesos de formación. La investigación educativa en México, 1992-2002. México: Consejo Mexicano de Investigación Educativa, (2005).

"Hábitos de los usuarios de Internet en México". Asociación Mexicana de Internet, AMIPCI. 2015. 15 de diciembre 2016.

https://www.amipci.org.mx/images/AMIPCI_HABITOS_DEL_INTERNAUTA_M EXICANO_2015.pdf

“Información Estadística Institucional”. Universidad Veracruzana. 2015. 20 de Noviembre 2016. http://www.uv.mx/informacion-estadistica/series-estadisticas-historicas/

López, Rocío. “Jóvenes universitarios: uso de las tecnologías digitales”. México. UNAM-Díaz de Santos, 2014.

López, Rocío y Anaya, Roberto. "Estudiantes universitarios interactuando en red: ¿nuevos escenarios de interacción, expresión y participación ciudadana?” [Versión electrónica] Revista Interamericana de Educación de Adultos, 1 (2016): 60-90.

Lópery López doi: https://doi.org/10.36799/el.v2i1.45 Volumen 2, número 1, Año 2017, ISSN: 2448-5942 
López, Rocío, Domínguez, Fernando y Quiñones, Fredy. "Estudiantes universitarios interactuando en red: el caso de la Universidad Veracruzana”. [Memoria electrónica] XIII Congreso Nacional de Investigación Educativa, COMIE, (2015).

López, Rocío y Domínguez, Fernando. "Uso de las redes sociales digitales entre los estudiantes de la Universidad Veracruzana”. VIII Seminario Regional (Cono Sur) ALAIC “Políticas, actores y prácticas de la comunicación: encrucijadas de la investigación en América Latina, (2015).

Ortiz, Gladys. "L@s jóvenes y su relación con la red internet: de la adicción al consumo cultural, Razón y Palabra". [Versión electrónica] Revista Electrónica en America Latina especializada en Comunicación, 78 (2012): 1-15

Ortiz, Gladys y López, Rocío. “Expresión, interacción y activismo social: hacia una construcción de escenarios digitales entre los jóvenes mexicanos.” En Valdés, Lucas. y Morales, Susana (comp.), Industrias culturales, medios y públicos: de la recepción a la apropiación. Argentina: Universidad Nacional de Córdoba, (2013).

"Redes sociales en México y Latinoamérica Estudio". Asociación Mexicana de Internet-AMIPCI. 2011. 5 de enero 2017. http://www.amipci.org.mx/temp/RedesSociales2011Final$\underline{0117676001317311680 O B . p d f}$

Sixto, José; Aguado, Nicolás y Riveiro, Reyes. "Presencia 2.0 de las pymes gallegas: niveles de participación y engagement con los usuarios” [versión electrónica] Revista Latina de comunicación social, 72 (2017): 47-68.

Tuñez, Miguel y Sixto, José. "Las redes sociales como entorno docente: análisis del uso de Facebook en la docencia universitario" [versión electrónica] Píxel-Bit revista de Medios y Educación, 41 (2012): 77-92.

Valerio, Gabriel; Leyva, Tania; Caraza, Ricardo y Rodríguez-Martínez, María. "Redes sociales en línea y la capacidad de memorización de los estudiantes universitarios”. [Versión electrónica] REDIE. Revista Electrónica de Investigación Educativa, 3 (2014): 118-128.

Lópery López doi: https://doi.org/10.36799/el.v2i1.45 Volumen 2, número 1, Año 2017, ISSN: 2448-5942 